\title{
Surface-induced alignment at model nematic interfaces
}

\author{
E. Martín del Río, ${ }^{*}$ M. M. Telo da Gama, and E. de Miguel* \\ Departamento de Física da Faculdade de Ciências e Centro de Física da Matéria Condensada, Universidade de Lisboa, \\ Avenida Prof. Gama Pinto 2, 1699 Lisboa Codex, Portugal \\ L. F. Rull \\ Departamento de Física Atómica, Molecular y Nuclear, Universidad de Sevilla, Apartado 1065, Sevilla 41080, Spain
}

(Received 30 June 1995)

\begin{abstract}
We have applied the generalized van der Waals theory to a model liquid crystal that includes, explicitly, all of the second-order terms in the spherical harmonic expansion of the anisotropic intermolecular potential. We have investigated the orientational order induced by each one of these terms, as well as the order resulting from the competition of various terms included in the potential. It was shown that, for appropriate choices of the relative strengths of the spherical harmonic coefficients, the theory is capable of accounting qualitatively for all the orientational effects observed at nematic interfaces, including tilted orientations. In particular, different molecular alignments at the nematic-vapor and nematic-isotropic interfaces of a given nematogen were described as the result of competing terms in the anisotropic interactions. Additionally, we have shown that temperaturedriven orientational transitions may occur in systems characterized by this type of interaction. For a given choice of parameters, we have also found an orientational transition, which is related to a wetting transition at the nematic-vapor interface. Finally, it was shown that complete wetting of the isotropic liquid-vapor interface by the nematic phase may be destroyed as a result of the competition between different terms in the potential. Similarly, a reentrant wetting transition at the nematic-vapor interface by the isotropic phase was found, as a result of this competition, at the nematic-vapor interface.
\end{abstract}

PACS number(s): $61.30 . \mathrm{Cz}, 61.30 . \mathrm{Gd}, 68.10 . \mathrm{Cr}, 68.45 . \mathrm{Gd}$

\section{INTRODUCTION}

The understanding of the interfacial properties of molecular liquids has long been recognized as a fundamental problem in the statistical physics of inhomogeneous systems. In the simplest case of coexisting isotropic phases, a planar interface breaks the translational symmetry of the system and induces, in general, a lower rotational symmetry at the interface. Several years ago, Gubbins [1] predicted a preferred molecular orientation at the liquid-vapor interface of fluids of nearly spherical molecules, such as $\mathrm{N}_{2}$ and $\mathrm{Cl}_{2}$, based on the results of perturbation theories and molecular dynamics simulations. These effects are, however, too small to be observed experimentally and of little practical relevance.

By contrast, surface-induced molecular alignment is expected to be important when liquid crystalline phases are involved and it is known to have a wide range of practical applications. The theoretical understanding of the anchoring mechanisms at liquid crystal surfaces and interfaces is still rather poor. This is due in part to the

\footnotetext{
*Permanent address: Departamento de Física Atómica, Molecular y Nuclear, Universidad de Sevilla, Apartado 1065, Sevilla 41080, Spain.
}

complexity of realistic liquid crystal interactions, which renders a theoretical description of nonuniform systems almost unfeasible. Additionally, the tensorial nature of the orientational order parameter is often exhibited by non-uniform nematogens (i.e., broken biaxial symmetry may spontaneously occur at interfaces) and thus even phenomenological theories are considerably harder to solve than similar Landau theories for systems with scalar order parameters.

For simplicity, we shall study translationally invariant phases only, i.e., isotropic liquid, vapor, and nematic phases. In the absence of surfaces or external fields, the average direction of molecular alignment in the bulk nematic is arbitrary. This is no longer the situation at the interface between the nematic and the vapor (or isotropic liquid). The translational symmetry of the system is now broken and the nematic director will be determined by the direction that minimizes the free energy of the inhomogeneous system. This effect is not expected to be universal, but seems to depend sensitively on the details of the intermolecular interactions [2].

This is indeed what has been observed experimentally. The preferential molecular orientation at the nematicisotropic $(N I)$ and nematic-vapor $(N V)$ interfaces was reported to be either perpendicular [3-5], parallel [6-8], or tilted [9-11], depending on the specific nematogen. In some cases, a temperature-dependent oblique tilt angle was also found $[4,6,12,13]$.

5028 (C) 1995 The American Physical Society 
Although various theoretical approaches have been used to date to describe orientational effects at nematic interfaces, a full understanding of the underlying mechanisms is still lacking. Moreover, some of these treatments yield somewhat contradictory results and thus the status of the models and/or of the approximations used in these studies is far from clear.

The first attempt to account for the existence of a preferred molecular orientation at the $N I$ interface was made by de Gennes [14]. By using a Landau-de Gennes expansion of the free energy ix powers of a single order parameter, both homeotrópic (perpendicular to the interface) and homogeneous planar alignments were obtained, depending on the sign of the square-gradient term (which is related to the elastic constants of the isotropic phase). Although several theories were developed following de Gennes's pioneering work, their phenomenological nature restricts their predictions to rather general qualitative trends. Furthermore, the connection between the expansion coefficients and the microscopic details of the molecular interactions is far from trivial, making it difficult to interpret the physical mechanisms that are responsible for the orientational order of a particular nematogen.

A different approach was followed by the so-called microscopic theories, which start by considering specific models for the intermolecular interactions (molecular shape, anisotropic attractive interactions, etc.) and then, based on a given theory, deduce the mechanisms that are ultimately responsible for the preferred interfacial alignment of a specific (model) nematogen. In line with bulk studies, two different models have been investigated in detail: an "Onsager-type" model, which takes into account solely the effects of anisotropic hard-core repulsions, and a "Maier-Saupe-type" model, which considers explicitly anisotropic interactions that are, on average, attractive. Some of these studies, however, may be considered as a combination of the two approaches, as will be discussed in the following paragraphs.

Most studies of the interfacial properties of liquid crystals based on hard-body models consider the molecular units to be spherocylinders. As far as the free nematic surface is concerned, it is a general finding that the hardcore repulsions favor perpendicular alignment at the interface $[15,16]$. Discrepancies, however, appear regarding the orientation at the $N I$ interface. While most studies report planar alignment [17-20], Holyst and Poniewersky [16] predict an oblique tilt angle of $60^{\circ}$, which was found to be independent of the molecular elongation, in agreement with experimental results for the 4-cyano-4'( $n$-alkyl)biphenyl (nCB) series [2]. This result was seriously questioned by Moore and McMullen [21], who found, in general, planar alignment, with the exception of systems with very small elongations where an oblique tilt angle was also obtained. More recently, however, Chen and Noolandi [20] suggested that the oblique tilt angle obtained in Refs. [16,21] is an artifact of the approximations used in the solution of the Onsager free-energy functional, in the limit of short length to breath ratios. Indeed, Chen and Noolandi [20] claimed that, within the Onsager approximation, a system of hard spherocylin- ders exhibits planar alignment at the $N I$ interface for all molecular elongations.

A rather different approach includes a number of theories that emphasize the interfacial ordering effects arising from the longer-ranged anisotropic attractive interactions. Many of these theories are based on highly idealized models and predict the same preferred orientation at the $N I$ and $N V$ interfaces, by contrast with the hardbody models and the experimental observations. Additionally, planar or perpendicular alignment is generally reported and thus no account of an oblique tilt angle seems to be given. An exception is the model studied by Kimura and Nakano, which is much more elaborate $[15,17]$. In these references the authors accounted for different orientations at the $N I$ and $N V$ interfaces of a given nematogen and predicted a (temperaturedependent) oblique tilt angle as a result of the competition between the repulsive and attractive interactions, for a range of model parameters.

More than a decade ago, one of us [22] developed a generalized van der Waals theory for nonuniform molecular fluids. This theory was applied to model nematic liquid crystals with anisotropic attractive interactions truncated at the Maier-Saupe level. No preferred orientation at the interface of this model was obtained, since the orientational and spatial dependences of the intermolecular interactions are decoupled in the Maier-Saupe potential. This theory was subsequently applied to a more realistic (although still highly idealized) model by Thurtell et al. [23] and Tjipto-Margo et al. [24] by including an extra term in the intermolecular potential, which couples the translational and rotational degrees of freedom. This extended model yields perpendicular alignment at the free nematic surface for prolate molecules, while planar alignment is predicted for oblate molecules. These results disagree with those obtained by Parsons [25] and by Harrowell and Oxtoby [26], who reported the opposite trend for systems of perfectly alligned ellipsoidal molecules.

In this paper we apply the generalized van der Waals theory to a liquid crystal model that includes, explicitly, all of the second-order terms in the spherical harmonic expansion of the anisotropic intermolecular potential. This model includes two terms that were not considered in closely related work [22-24]. We have investigated the orientational order induced by each one of these terms as well as the order resulting from the competition of the various terms included in the intermolecular potential.

In Sec. II we summarize the theory used in this work applied to our model liquid crystal. In Sec. III we present and discuss the results for the orientational order at the $N V$ and $N I$ interfaces. It is shown that, for appropriate choices of the relative strengths of the spherical harmonic coefficients of the intermolecular potential, the theory is capable of accounting qualitatively for all the orientational effects at nematic interfaces found in experiments. The wetting behavior of these systems, at the triple point, is also investigated for particular combinations of the intermolecular parameters. Finally, we summarize our results in Sec. IV and compare them with other theoretical works and available experimental data. 


\section{THEORY}

We start by summarizing the mean-field theory for a nonuniform molecular fluid, which was proposed in the context of liquid crystal interfaces more than 10 years ago [22]. Applications of this theory to fluid and solidfluid interfaces of model nematogens have been reviewed recently [27]. For convenience we will consider an open system at temperature $T$ and chemical potential $\mu$, in a volume $V$. In the absence of external fields, the grand potential $\Omega$ of the inhomogeneous system is the minimum of the functional

$$
\begin{aligned}
\Omega[\rho(\vec{r}, \omega)]= & \int d \vec{r} f_{\mathrm{ref}}(\vec{r})-\mu \int d \vec{r} d \omega \rho(\vec{r}, \omega) \\
& +\frac{1}{2} \iint_{V} d \vec{r} d \vec{r}^{\prime} d \omega d \omega^{\prime} u_{p}\left(\vec{r}, \omega ; \vec{r}^{\prime}, \omega^{\prime}\right) \\
& \times \rho(\vec{r}, \omega) \rho\left(\vec{r}^{\prime}, \omega^{\prime}\right)
\end{aligned}
$$

where $\rho(\vec{r}, \omega)$ is the density-orientational profile, which is a function of the molecular center of mass $\vec{r}$, and of the molecular orientation $\omega$ for rigid molecules. The molecular interactions, which are supposed to be pairwise additive, are split into a short-ranged (generally repulsive) reference potential and a longer-ranged (usually attractive) perturbation. The free energy of the reference system is written within a local density approximation; following previous work [22] we assume the reference system to be a fluid of hard particles, i.e., $f_{\text {ref }}(\vec{r})$ is the Helmholtz free-energy density of a uniform hard-core fluid with density $\rho_{0}(\vec{r})=\int d \omega \rho(\vec{r}, \omega)$ and orientational distribution function $f(\vec{r}, \omega)=\rho(\vec{r}, \omega) / \rho_{0}(\vec{r})$. The contribution to the free energy from the long-ranged (attractive) interactions is given by a mean-field average of the corresponding pair potential $u_{p}$.

In line with previous work [22-24], we consider the simplest possible model characterized by spherical hard cores and linear molecules. In this case, the free-energy density of the reference fluid is greatly simplified and can be written as [22]

$$
f_{\mathrm{ref}}(\underline{r})=f_{\mathrm{HS}}(\vec{r})+\rho_{0}(\vec{r}) k_{B} T\langle\ln 4 \pi f(\vec{r}, \omega)\rangle,
$$

where $f_{\mathrm{HS}}$ is the free-energy density of a system of hard spheres (given for consistency with earlier results by the Percus-Yevick approximation), $k_{B}$ is Boltzmann's constant, and $\langle A(\vec{r}, \omega)\rangle=\int d \omega f(\vec{r}, \omega) A(\vec{r}, \omega)$ is a weighted angular average over the orientational distribution function. The last term in (2) accounts for the loss of orientational entropy of the reference system, within a random mixing (mean-field) approximation.

The Euler-Lagrange equations for $\rho_{0}(\vec{r})$ and $f(\vec{r}, \omega)$ are obtained by minimization of Eq. (1) and are written in terms of the effective potential as

$$
\begin{gathered}
\mu=\mu_{\mathrm{HS}}(\vec{r})+k_{B} T\langle\ln 4 \pi f(\vec{r}, \omega)\rangle+\left\langle u_{\mathrm{eff}}(\vec{r}, \omega)\right\rangle, \\
f(\vec{r}, \omega)=\frac{\exp \left[-u_{\mathrm{eff}}(\vec{r}, \omega) / k_{B} T\right]}{\int d \omega \exp \left[-u_{\mathrm{eff}}(\vec{r}, \omega) / k_{B} T\right]},
\end{gathered}
$$

where $\mu_{\mathrm{HS}}(\vec{r})$ is the chemical potential of a uniform sys- tem of hard spheres and the effective potential $u_{\mathrm{eff}}(\vec{r}, \omega)$ acting on the reference system is given by

$u_{\mathrm{eff}}(\vec{r}, \omega)=\int d \vec{r}^{\prime} d \omega^{\prime} u_{p}\left(\vec{r}, \omega ; \vec{r}^{\prime}, \omega^{\prime}\right) \rho_{0}\left(\vec{r}^{\prime}\right) f\left(\vec{r}^{\prime}, \omega^{\prime}\right)$

Equations (3a) and (3b) are solved numerically once the intermolecular potential $u_{p}$ is specified. The (longranged) perturbation potential studied in this work is a generalization of the potential used previously [22-24] and it includes all the invariants in the spherical harmonic expansion of an arbitrary anisotropic potential, with inversion symmetry, up to second-order. Thus two different terms, neglected in Refs. [23,24], are included explicitly in our model. We note that a similar potential was analyzed by Sullivan and Tjipto-Margo [28] within a simplified version of the theory described in this paper. We will return to this point in Sec. III, where we discuss our results for the interfaces of liquid crystals described by this model.

The general expansion for an arbitrary anisotropic intermolecular potential is written as

$$
\begin{aligned}
u_{\mathrm{ani}}\left(\vec{r}, \omega_{1}, \omega_{2}\right)= & \sum_{l_{1}, l_{2}, l} \sum_{m_{1}, m_{2}, m} u\left(l_{1} l_{2} l ; r\right) \\
& \times C\left(l_{1} l_{2} l ; m_{1} m_{2} m\right) \\
& \times Y_{l_{1} m_{1}}\left(\omega_{1}\right) Y_{l_{2} m_{2}}\left(\omega_{2}\right) Y_{l m}^{*}\left(\omega_{12}\right)
\end{aligned}
$$

where $r=\left|\vec{r}_{2}-\vec{r}_{1}\right|$ is the intermolecular distance, $C\left(l_{1} l_{2} l ; m_{1} m_{2} m\right)$ is a Clebsch-Gordan coefficient, and $Y_{l m}(\omega)$ is a spherical harmonic, all in the notation of Gray and Gubbins [29]. Our model for the anisotropic potential amounts to keeping terms in the expansion Eq. (5), with $l_{1}=0,2$ and $l_{2}=0,2$ only. In addition, we will assume an $r$ dependence for the expansion coefficients $u\left(l_{1} l_{2} l ; r\right)$, which is appropriate to dispersion interactions, but we will consider the signs and magnitudes of the various terms as free parameters. These can then be written as

$$
\begin{gathered}
u(000 ; r)=-(4 \pi)^{3 / 2} A\left(\frac{\sigma}{r}\right)^{6}, \\
u(220 ; r)=-\frac{(4 \pi)^{3 / 2}}{\sqrt{5}} B\left(\frac{\sigma}{r}\right)^{6}, \\
u(202 ; r)=u(022 ; r)=\frac{(4 \pi)^{3 / 2}}{5} C\left(\frac{\sigma}{r}\right)^{6}, \\
u(222 ; r)=(4 \pi)^{3 / 2} \sqrt{\frac{7}{10}} D\left(\frac{\sigma}{r}\right)^{6}, \\
u(224 ; r)=\frac{(4 \pi)^{3 / 2}}{3} \sqrt{\frac{7}{10}} E\left(\frac{\sigma}{r}\right)^{6}
\end{gathered}
$$

if $r>\sigma$ and $u\left(l_{1} l_{2} l ; r\right)=0$ if $r<\sigma$, where $\sigma$ is the diameter of the spherical hard core. $A, B, C, D$, and $E$ are arbitrary constants. In what follows, each term of 
the anisotropic potential is denoted by the corresponding set of indices $\left(l_{1} l_{2} l\right)$. We note that for particular combinations of the constants $A-E$, the potential given by Eqs. (5) and (6) corresponds to well defined microscopic models of the anisotropic interactions. We mention two of these that are relevant in studies of liquid crystals: anisotropic dispersion interactions [29] and a spherical harmonic expansion of the Gay-Berne potential [30].

We recall at this point that the thermodynamic properties of the uniform bulk phases, within the mean-field theory discussed in this section, depend only on the values of the parameters $A$ (which sets the energy scale) and $B$ (which determines the isotropic-nematic-vapor triple point) [23]. Stability of the isotropic liquid-vapor and nematic-isotropic liquid transitions requires that $A$ and $B$ be positive (respectively). The reader is referred to Refs. $[22,31]$ where full details of the bulk phase diagrams, including a diagram characterized by a nematic-nematic critical point [31], may be found. In this study we do not consider bulk phases with broken translational invariance (e.g., smectic) and thus the interaction parameters that may be used in Eqs. (6) are somewhat restricted in order to guarantee the stability of the nematic phase.

We consider a planar interface in the $x-y$ plane. Without loss of generality, the nematic director (average direction of alignment of the molecules) is assumed to lie in the $x-z$ plane due to the rotational invariance of the system in the interfacial plane. The orientational structure is then characterized by three independent order-parameter profiles [22], defined as angular averages of linear combinations of second-order spherical harmonics:

$$
\begin{gathered}
\eta(z)=\int d \omega f(z, \omega) P_{2}(\cos \theta) \\
\nu(z)=\int d \omega f(z, \omega) \sin 2 \theta \cos \phi \\
\sigma(z)=\int d \omega f(z, \omega) \sin ^{2} \theta \cos 2 \phi .
\end{gathered}
$$

$\eta=1$ when there is full alignment of the molecules perpendicular to the interface, $\sigma=1$ when the molecules are fully aligned along the $x$ axis, and $\nu=1$ when all the molecules are aligned parallel to the bisector of the $x-z$ plane.

In the following sections, we will take $A$ and $\sigma$ (the hard-core diameter) as units of energy and length, respectively. In addition we simplify the notation by taking Boltzmann's constant $k_{B}=1$. In terms of the order parameters defined through Eqs. (7a)-(7c), the effective potential Eq. (4) reads

$$
\begin{aligned}
u_{\mathrm{eff}}(z, \omega)= & \int d z^{\prime} \rho_{0}\left(z^{\prime}\right)\left\{-u_{0}\left(\left|z-z^{\prime}\right|\right)+C u_{2}\left(\left|z-z^{\prime}\right|\right)\left[\eta\left(z^{\prime}\right)+P_{2}(\cos \theta)\right]\right. \\
& +\eta\left(z^{\prime}\right) P_{2}(\cos \theta)\left[-B u_{0}\left(\left|z-z^{\prime}\right|\right)-D u_{2}\left(\left|z-z^{\prime}\right|\right)+3 E u_{4}\left(\left|z-z^{\prime}\right|\right)\right] \\
+ & \frac{3}{4} \nu\left(z^{\prime}\right) \sin 2 \theta \cos \phi\left[-B u_{0}\left(\left|z-z^{\prime}\right|\right)-\frac{D}{2} u_{2}\left(\left|z-z^{\prime}\right|\right)-2 E u_{4}\left(\left|z-z^{\prime}\right|\right)\right] \\
& \left.+\frac{3}{4} \sigma\left(z^{\prime}\right) \sin ^{2} \theta \cos 2 \phi\left[-B u_{0}\left(\left|z-z^{\prime}\right|\right)+D u_{2}\left(\left|z-z^{\prime}\right|\right)+\frac{E}{2} u_{4}\left(\left|z-z^{\prime}\right|\right)\right]\right\},
\end{aligned}
$$

where the functions $u_{l}(z)$ are given by

$$
\begin{gathered}
u_{0}(z)= \begin{cases}\pi / 2 & \text { if }|z|<1 \\
\pi /\left(2 z^{4}\right) & \text { if }|z|>1,\end{cases} \\
u_{2}(z)= \begin{cases}\frac{\pi}{2}\left(z^{2}-1 / 2\right) & \text { if }|z|<1 \\
\pi /\left(4 z^{4}\right) & \text { if }|z|>1,\end{cases} \\
u_{4}(z)= \begin{cases}\frac{\pi}{4}\left(35 / 8 z^{4}-5 z^{2}+3 / 4\right) & \text { if }|z|<1 \\
\pi /\left(32 z^{4}\right) & \text { if }|z|>1 .\end{cases}
\end{gathered}
$$

The solution of the Euler-Lagrange equations [Eqs. (3)] is now reduced to the solution of the one-dimensional integral equation [Eq. (3a)] for $\rho_{0}(z)$ and the consistency relations [Eqs. (7)] for the orientational order parameters. We follow the method used in Ref. [24] to solve these equations numerically, since it has proved much more accurate [24] than the simpler method used in the earlier calculations $[22,23]$. Our results, however, differ slightly from those of Ref. [24] (typically 1 part in $10^{3}$ ) due to a small error that has been found subsequently in the latter [32].

\section{RESULTS}

\section{A. Orientational order at the interface}

As we have mentioned, the fluid (nematic-isotropicvapor) phase diagram is independent of the parameters $C, D$, and $E$ within the mean-field approximation discussed in Sec. II; however, these terms play a significant role in spatially inhomogeneous situations (both at interfaces and in bulk modulated phases [33]). In our cal- 
culations, we have set $B=0.3$, in line with earlier work [22-24], and varied the other parameters in $u_{\text {ani }}(C, D$, and $E)$. For this value of $B$, the phase diagram exhibits three uniform fluid phases [22]: nematic $(N)$, isotropic liquid $(I)$, and vapor $(V)$. These phases coexist simultaneously at the triple point, which occurs at a temperature $T_{\mathrm{tr}}=0.218156$. At lower temperatures $T<T_{\mathrm{tr}}$ the vapor coexists with an ordered $N$ phase and at higher temperatures $T>T_{\mathrm{tr}}$ it coexists with a disordered $I$ liquid.

If only the $l=0$ terms are included in the intermolecular potential $u_{\text {ani }}$ [Eq. (5)], no preferred orientation at the interface is obtained [22]. This arises from the fact that if (220) is the only anisotropic interaction, the orientational and translational degrees of freedom are decoupled, with the result that the interface is rotationally invariant. We refer to this model as the Maier-Saupe model.

The effect of including the term (202) in $u_{\text {ani }}$, [Eq. (5)] $(C \neq 0$ and $D=E=0)$ was analyzed by Thurtell et al. [23] and by Tjipto-Margo et al. [24]. Defining the tilt angle $\Psi$ as the angle between the nematic director and the normal to the interface ( $z$ axis), Thurtell et al. [23] found $\Psi=0^{\circ}$ (homeotropic alignment) for $C>0$ (prolate molecules) and $\Psi=90^{\circ}$ (homogeneous planar alignment) for $C<0$ (oblate molecules) at both the $N V$ and $N I$ interfaces. Although the tilt at the $N V$ interface appears to be either $0^{\circ}[3-5]$ or $90^{\circ}[6,7]$, in agreement with the results of this model, no oblique tilt angle, as observed at the $N I$ interface for a series of nematogens $[10,11]$, is obtained. More terms in the potential seem to be required in order to account for the whole range of experimental results. In this paper we go one step in that direction by analyzing, in turn, the various anisotropic terms in the potential of Eqs. (5) and (6).

We have studied the preferred interfacial orientation induced by the terms (with $l \neq 0$ ) in $u_{\text {ani }}$, at the nematicvapor interface, at a fixed temperature $T=0.175$ (which is far from the triple point). In the absence of competition between terms of the intermolecular potential, the preferred molecular orientation (equilibrium tilt angle) is the same at the $N I$ and $N V$ interfaces. This is not true for real nematogens [2], which suggests that in these systems such a competition may be relevant, resulting in different tilt angles at the $N I$ and $N V$ interfaces. We will return to this point in Sec. III C.

In order to solve Eqs. (3), the orientation of the bulk nematic director is required. Since the bulk phase is rotationally invariant, at equilibrium, the bulk director is determined by the interfacial tilt angle. Thus it may be difficult to obtain convergent numerical solutions starting from an arbitrary direction of the bulk nematic director. In order to overcome this difficulty, we have used an ansatz proposed by Tarazona and Evans [34] in the context of wetting phenomena. We start by fixing the angle $\Psi$ between the bulk nematic director and the normal to the interface and consider an initial guess for the densityorientational profile compatible with the given $\Psi$; then the integral equations (3) are iterated for a fixed number of times $M$ ( $M$ depends on the proximity to the triple point under wetting conditions, but it is otherwise arbitrary). The above process is repeated for different values of $\Psi$. The minimum of the excess grand potential as a function of $\Psi$ yields an estimate of the equilibrium tilt angle. The equilibrium tilt is now calculated by solving Eqs. (3) for different values of $\Psi$ in the neighborhood of the minimum, until a predetermined convergence criterium is satisfied. Since the tilt angle may vary slowly at the interface, we use the bulk value of $\Psi$ as the equilibrium tilt angle. The value of the excess grand potential corresponding to the equilibrium tilt angle is the equilibrium surface tension.

Let us consider first the $N V$ interface. We start by choosing $C= \pm 0.3(D=E=0)$. In agreement with previous work $[23,24]$, we find that the surface tension is lowest when $\Psi=0^{\circ}\left(90^{\circ}\right)$ for $C>0(<0)$ (see Fig. 1). A similar analysis of the (222) term was carried out by setting $D= \pm 0.3$ (with $C=E=0$ ). The results [Fig. 1(a)] indicate that the (222) term yields an equilibrium tilt an-
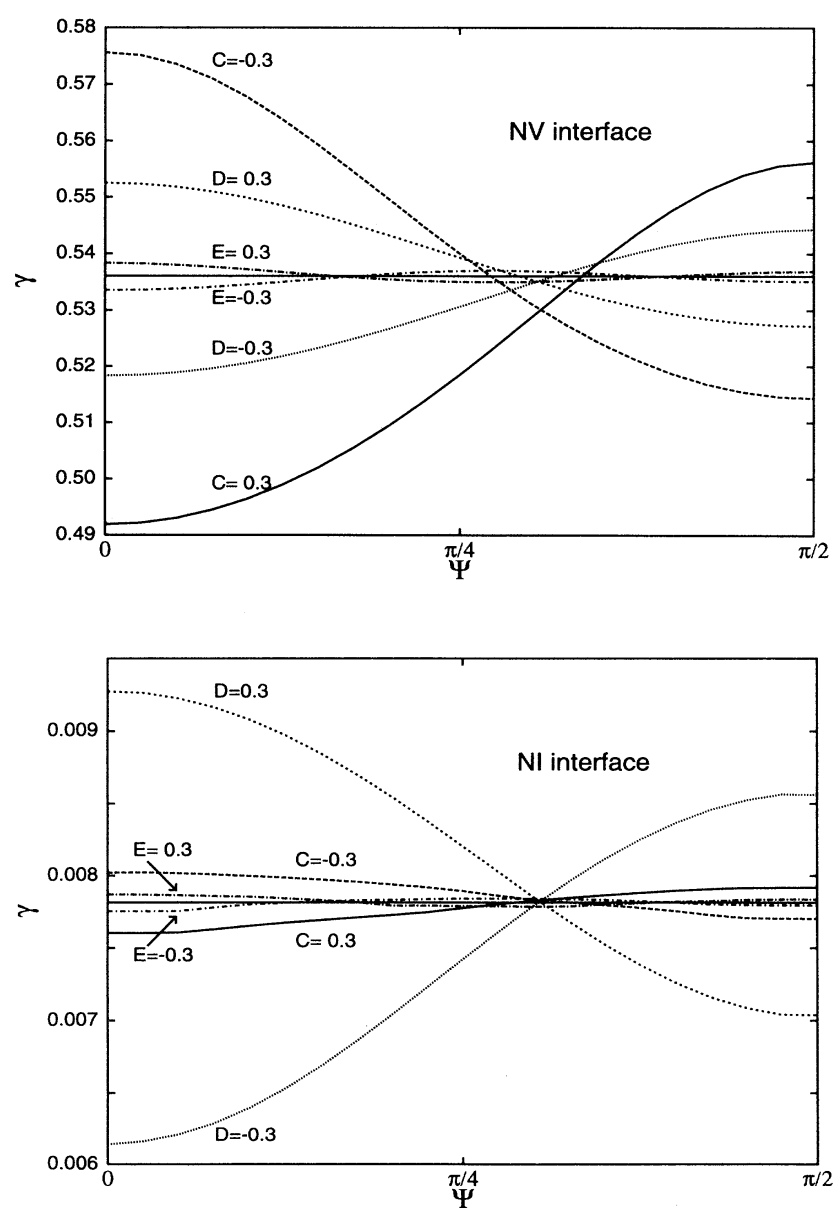

FIG. 1. Excess grand potential $\gamma$ (in units of $A \sigma^{-2}$ ) as a function of the orientation $\Psi$ of the bulk nematic director for each of the terms in $u_{\text {ani }}$ [Eq. (5)]. The straight line is the surface tension of the Maier-Saupe nematogen. The other curves are the surface tensions when one of the other terms is included: (202) with $C= \pm 0.3,(222)$ with $D= \pm 0.3$, and (224) with $E= \pm 0.3$, respectively. (a) $N V$ interface $(T=0.175)$ and (b) $N I$ interface $(T=0.225)$. 
gle that is opposite the tilt due to the (202) term, i.e., when $D>0$ the molecules are aligned parallel to the interface while for $D<0$ the molecules are normal to the interface. The behavior of the surface tension (strictly speaking, we should reserve the term surface tension for the minimum of the excess grand potential, but following current practice we speak of a surface tension as a function of $\Psi$ ) with $\Psi$ is monotonic in both cases [see Fig. 1(a)]. The change in the surface tension of the $N V$ interface characterized by the Maier-Saupe potential (220) (straight line in Fig. 1) due to the inclusion of the (202) term is significantly larger than that due to the inclusion of a (222) term of similar magnitude.

Finally, the effects of the (224) term (proportional to $E)$ are particularly interesting. For $E>0$, the surface tension $\gamma$ is lowest when $\Psi \approx 49.5^{\circ}$. This value is very close to that given by a first-order perturbation expansion in terms of $E$ (see the next paragraph) and to the value given by a sharp-kink approximation of this mean-field theory [28]. $\Psi=0^{\circ}$ corresponds to the maximum of the surface tension while $\Psi=90^{\circ}$ is a local maximum (Fig. 1). In contrast, when $E<0$, the equilibrium tilt angle is $\Psi=0^{\circ}$; in this case $\Psi \approx 49.5^{\circ}$ corresponds to the maximum of the surface tension and $\Psi=90^{\circ}$ corresponds to a local minimum.

The change in the surface tension of the Maier-Saupe nematogen [straight line in Fig. 1(a)] due to the inclusion of the (224) term alone is much smaller than the changes due to either the (202) or the (222) term discussed in the preceding paragraph. We note that, although the preferred orientations at the $N I$ interface, induced by the various terms with $l \neq 0$, are the same as those found at the $N V$ interface, the relative change in the $N I$ surface tension is largest when the (222) term is included [see Fig. 1(b)].

Although we do not expect realistic values of $E$ or $D$ to be larger than $A$, our theory breaks down for $D \geq 1.2$ or $E \geq 0.9$, for a system with $B=0.3$ and $C=0$. This is signaled by the appearance of "structured" phases at the nematic free surface when $D$ or $E$ reaches those values. Whether these phases are real (for the given potentials) or are an artifact of the mean-field approximation requires further study. In this paper we have restricted our attention to values of $D$ and $E$ that are much smaller than those corresponding to the instability.

The results of Fig. 1 can be understood using a perturbation expansion of the surface tension about the MaierSaupe nematogen $(C=D=E=0)$ similar to that used in Ref. [24]. Following Tijpto-Margo et al., the surface tension difference between a system with nonzero $C(D=E=0)$ and a reference Maier-Saupe system is written, to first order in $C$, as

$$
\gamma-\gamma^{(0)}=-C J_{C} P_{2}(\cos \Psi),
$$

where $J_{C}$ is a positive number and $P_{2}(x)$ is the secondorder Legendre polynomial. As pointed out in [24], Eq. (12) implies that $\gamma$ is minimized by $\Psi=0^{\circ}$ when $C>0$ and by $\Psi=90^{\circ}$ when $C<0$.

Similarly we take $\gamma$ to be the surface tension of a system with nonzero $D(C=E=0)$ and find, to first order in $D$,

$$
\gamma-\gamma^{(0)}=\frac{1}{2} D J_{D} P_{2}(\cos \Psi)
$$

where $J_{D}$ is a constant given by

$$
\begin{aligned}
J_{D}= & \int d z_{1} \frac{d}{d z_{1}}\left[\rho^{(0)}\left(z_{1}\right) \eta_{I}^{(0)}\left(z_{1}\right)\right] \\
& \times \int d z_{2} \frac{d}{d z_{2}}\left[\rho^{(0)}\left(z_{2}\right) \eta_{I}^{(0)}\left(z_{2}\right)\right] G_{2}\left(\left|z_{1}-z_{2}\right|\right) .
\end{aligned}
$$

In the above expression, $\rho^{(0)}(z)$ is the density profile of the reference system, $\eta_{I}{ }^{(0)}(z)$ is the "intrinsic" uniaxial order parameter defined in [24], and $G_{2}(z)$ is a positive, monotonically decaying function of $z$ [see Eq. (A7) in Ref. [24]]. As both $\rho^{(0)}(z)$ and $\eta_{I}^{(0)}(z)$ are also monotonic functions, $J_{D}$ is always positive. From Eq. (13) it follows that $\gamma$ is minimized when $\Psi=90^{\circ}\left(0^{\circ}\right)$ if $D>0$ $(<0)$.

Finally, the difference between the surface tension of a system with $E \neq 0(C=D=0)$ and that of the reference Maier-Saupe system is written to first order in $E$ as

$$
\gamma-\gamma^{(0)}=-\frac{1}{2} E J_{E}\left[3 P_{2}{ }^{2}(\cos \Psi)-\frac{3}{2} \sin ^{2} 2 \Psi+\frac{3}{8} \sin ^{4} \Psi\right]
$$

where $J_{E}$ is a constant given by

$$
\begin{aligned}
J_{E}= & \int d z_{1} \frac{d}{d z_{1}}\left[\rho^{(0)}\left(z_{1}\right) \eta_{I}^{(0)}\left(z_{1}\right)\right] \\
& \times \int d z_{2} \frac{d}{d z_{2}}\left[\rho^{(0)}\left(z_{2}\right) \eta_{I}^{(0)}\left(z_{2}\right)\right] G_{4}\left(\left|z_{1}-z_{2}\right|\right)
\end{aligned}
$$

and $\rho^{(0)}(z)$ and $\eta_{I}^{(0)}(z)$ have the same meaning as before. The function $G_{4}(z)$ is the solution of the equation $d^{2} G_{4}(z) / d z^{2}=u_{4}(z)$. Using $u_{4}(z)$ given by Eq. (11), it follows that

$$
G_{4}(z)=\frac{\pi}{192}\left\{\begin{array}{cl}
\left(7 z^{6}-20 z^{4}+18 z^{2}-4\right) & \text { if }|z|<1 \\
1 / z^{2} & \text { if }|z|>1
\end{array}\right.
$$

From the behavior of $\rho^{(0)}(z)$ and $\eta_{I}{ }^{(0)}(z)$ [24], it follows that $\frac{d}{d z}\left[\rho^{(0)}(z) \eta_{I}{ }^{(0)}(z)\right]$ is nonzero in the interfacial region only; this implies that the main contribution to $J_{E}$ arises from small values of $\left|z_{1}-z_{2}\right|$. In this region $(z<0.5)$, the function $G_{4}(z)$ is negative and, as a consequence, Eq. (15) implies that $\gamma$ is minimized by $\Psi \approx 49^{\circ}$ when $E>0$ and by $\Psi=0^{\circ}$ when $E<0$. As the contribution to the expansion coefficient $u(224 ; r)$ arising from anisotropic dispersion interactions is always negative [29] $(E<0)$, these results indicate, in agreement with those of Refs. $[28,30]$, that this contribution cannot account for an oblique tilt angle at the interface. On the other hand, an electrostatic quadrupole-quadrupole contribution is indeed positive and, as pointed out by Sullivan and Tjipto-Margo $[28,30]$, this interaction may be relevant in stabilizing an oblique tilt angle as observed at the $N I$ interfaces of many nematogens [9-11]. 


\section{B. Competing surface orientations and tilt angle transitions}

Since the (202) and (222) terms compete as far as the alignment at the interface is concerned, orientational transitions may occur as the relative magnitude of these terms is changed. We have looked for orientational transitions by taking $C$ constant and varying $D$ (with $C D>0)$. We have set $C=0.3$ and studied the orientation at the $N V$ interface at $T=0.175$, as $D$ (with $E=0$ ) is increased from 0 . An orientational transition was found to occur for a value of $D$ in the range 0.70 0.75. In systems with $D<0.70$ the tilt angle is $\Psi=0^{\circ}$ while for $D>0.75$ the equilibrium tilt angle is $\Psi=90^{\circ}$. The transition is of first order, as indicated by the presence of an energy barrier in Fig. 2. We note that, close to the transition, the molecular orientation at the interface is neither perpendicular nor parallel. The tilt angle has a value that is rather close to $0^{\circ}$ or $90^{\circ}$ (see Fig. 2). We have not attempted to calculate the exact value of $D$ at which the orientational transition takes place. The transition occurs in a region where the linear terms of the perturbation expansion cancel out and thus a perturbation theory for the the excess grand potential requires the inclusion of higher-order terms. We stress that this competition does not yield equilibrium tilt angles of the order of $45^{\circ}$, as observed experimentally; indeed, within our model, these values of the tilt can only be obtained by inclusion of (224) terms in the intermolecular potential.

Similar transitions also occur at the $N I$ interface. For a system with $C=0.3$ (and $E=0$ ), at a fixed temperature $T=0.261$, we have found that $\Psi=0^{\circ}$ for $D<0.01$ and $\Psi=90^{\circ}$ for $D>0.02$. Note that the reverse transition takes place in a system characterized by symmetrical values of $C$ and $D$.

Sullivan and Tijpto-Margo [28] have studied the same

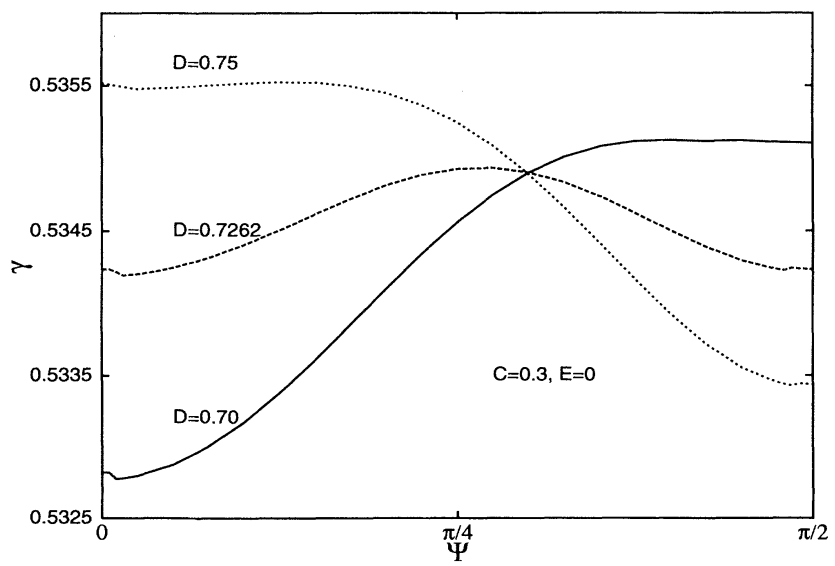

FIG. 2. Excess grand potential $\gamma$ at the $N V$ interface $(T=0.175)$ as a function of the orientation $\Psi$ of the bulk nematic director for $C=0.3, E=0$, and different values of $D$, close to the orientational transition from $\Psi_{\text {eq }} \approx 0^{\circ}$ to $\Psi_{\mathrm{eq}} \approx 90^{\circ}$. model potential using a mean-field theory. Rather than solving numerically the resulting integral equations, they have obtained analytical results by resorting to a sharpkink approximation for the density-orientational profile. Within this approximation a first-order transition between $\Psi=0^{\circ}$ and $\Psi=90^{\circ}$ takes place at the $N V$ interface, at $T=0.175$, for a system with $C=0.3$ and $D=0.7536$, which is close to the range of $D$ obtained from the full numerical solution. Similarly, within the sharp-kink approximation we estimate the transition at the $N I$ interface, at $T=0.261$, to occur for a system with $C=0.3$ and $D=0.0137$. The agreement with the full numerical results is somewhat surprising, since the sharpkink approximation is expected to be less reliable for the $N I$ interface. Note, however, that the relative range of $D$ 's estimated using the full mean-field theory is much larger for the transition at the $N I$ interface. Qualitative discrepancies have also been found. The first is the fact that the full mean-field theory predicts a transition to occur between two tilted orientations rather than between perpendicular and planar alignment. The second concerns the order of the transition when temperaturedriven orientational transitions are considered for fixed values of the parameters. This point will be further discussed in Sec. IIID.

\section{Competing surface orientations and tilt angles at the $N V$ and $N I$ interfaces}

It has been found experimentally [2] that for most systems, the orientation at the $N V$ interface differs from that at the $N I$ interface. This behavior may be described with the present theory, by including more than one term with $l \neq 0$ in the anisotropic intermolecular potential. Following our previous arguments, an appropriate combination of the parameters $C$ and $D$ may be chosen in order to obtain different molecular orientations at the $N V$ and $N I$ interfaces. If we take $C D>0$, the (202) and (222) terms compete and may yield different orientations at the $N V$ and $N I$ interfaces; when $D / C \approx 1$, the molecular alignment at the $N I$ interface is dictated by the (222) term (more precisely by the sign of $D$ ) and the orientation at the $N V$ interface is determined by the (202) term, i.e., by the sign of $C$.

In Fig. 3 we have plotted the density and orderparameter profiles at the $N I$ interface $(T=0.225)$ and at the $N V$ interface $(T=0.218)$ for a system with $C=D=0.3$ and $E=0$. This choice of parameters may be used to describe the experimental observations of 4-methoxybenzilidene- $4^{\prime}-(n$-butyl)aniline (MBBA) molecules close to the triple point: homeotropic alignment $\left(\Psi=0^{\circ}\right)$ at the $N V$ interface [12,13] and homogeneous planar alignment $\left(\Psi=90^{\circ}\right)$ at the $N I$ interface [8]. Note that while the $N V$ interface is uniaxial, the $N I$ interface is biaxial and two order parameters are required to describe the interfacial order.

We note that it is not necessary to take $D / C \approx 1$ in order to obtain different orientations at the $N I$ and $N V$ interfaces. For example, by taking $C=0.3(-0.3)$ and $0.02 \leq D \leq .7(-0.7 \leq D \leq-0.02)$ we find $\Psi=0^{\circ}\left(90^{\circ}\right)$ 
at the $N V$ interface and $\Psi=90^{\circ}\left(0^{\circ}\right)$ at the $N I$ interface

In Sec. III A it was pointed out that the (224) term has a relatively weak influence (compared with the other two terms with $l \neq 0$ ) in determining the interfacial ordering. Consequently, we have found oblique tilt angles, for positive $E$, when $C$ and $D$ are such that the contributions of the (202) and (222) terms nearly cancel. The value of $D / C$ required for this to occur at the $N I$ interface is, in general, much smaller than at the $N V$ interface (two orders of magnitude, for a system with $C=0.3$ ). Thus, within our model, it is not possible to find oblique tilted angles at both interfaces for a given nematogen.

We have chosen $C=0.3, D=0.01$, and $E=0.5$ to describe qualitatively the molecular orientation at the interfaces of nematogens in the $n C B$ series. In these systems, the alignment is found to be perpendicular at the $N V$ interface [3-5] while it is oblique at the $N I$ interface, with a tilt angle ranging from $48.5^{\circ}$ (in $8 \mathrm{CB}$ ) to $64.5^{\circ}$ (in
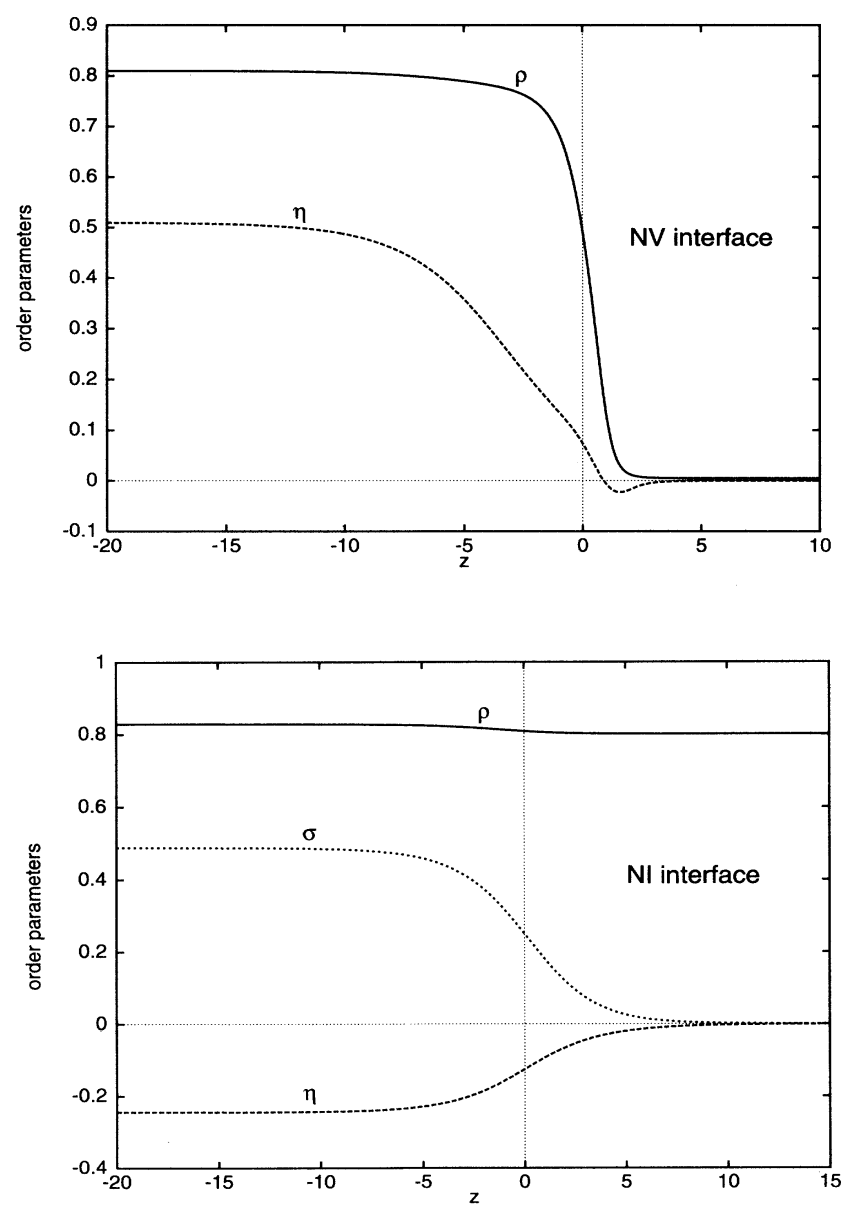

FIG. 3. Density (in units of $\sigma^{3}$ ) and order-parameter profiles for a system with $C=D=0.3$ and $E=0$. (a) $N V$ interface $(T=0.218)$. The equilibrium tilt angle is $\Psi_{\text {eq }}=0^{\circ}$ (as for MBBA close to the triple point $[12,13]$ ); the interface is uniaxial. (b) $N I$ interface $(T=0.225)$. The equilibrium tilt angle is $\Psi_{\text {eq }}=90^{\circ}$ (as for MBBA [8]); the interface is biaxial. $z$ coordinate is in units of $\sigma$.
6CB) $[10,11]$. In Fig. 4 we have plotted the density and order-parameter profiles for this model nematogen at the $N V(\mathrm{~T}=0.211)$ and $N I(T=0.225)$ interfaces. The $N V$ interface is uniaxial with $\eta$ the only nonzero order parameter, while the $N I$ interface is tilted biaxial characterized by three orientational order parameters.

\section{Temperature-driven tilt angle transitions}

Experiments on MBBA and 4-(n-ethoxy)benzilidene$4^{\prime}$ - $(n$-butyl $)$ aniline $[6,12,13]$ indicate that the molecular alignment at the $N V$ interface changes continuously from tilted to perpendicular orientation at a certain temperature $T_{0}$ slightly below the triple point. We have found
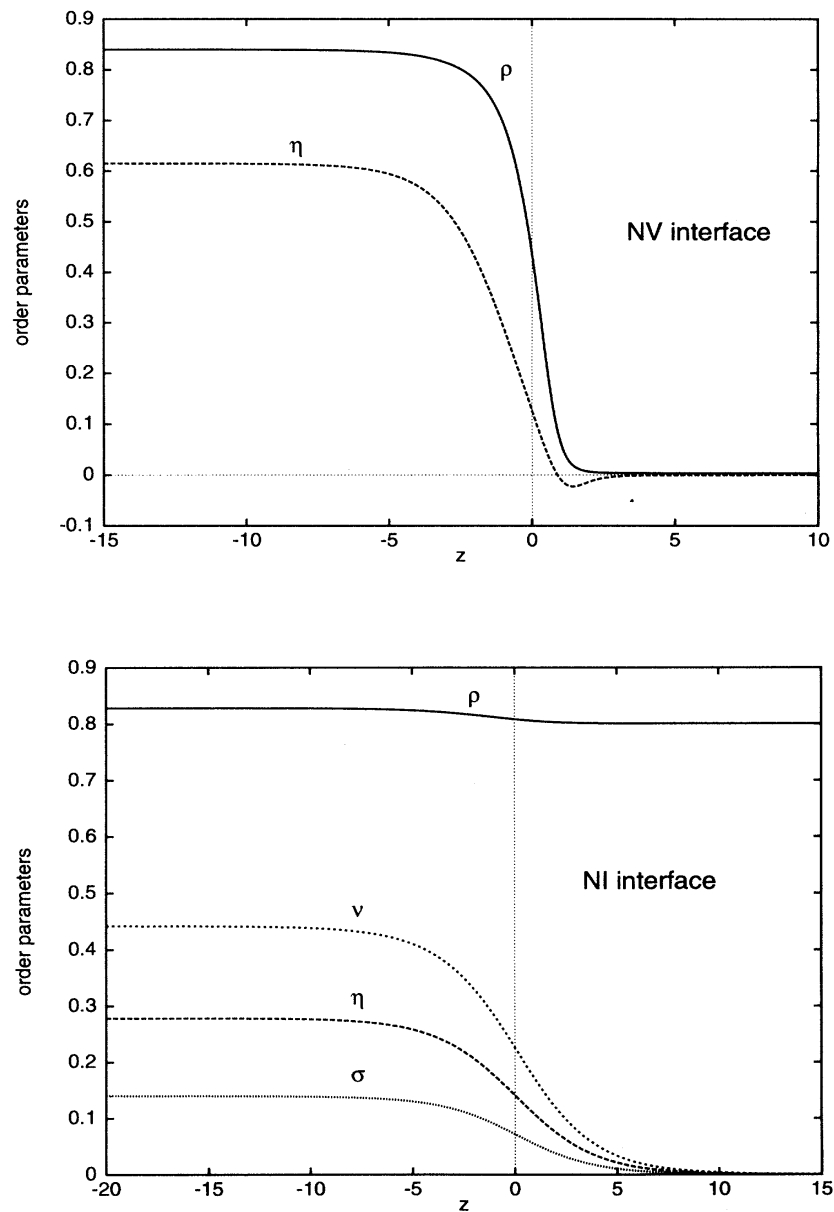

FIG. 4. Density and order-parameter profiles for a system with $C=0.3, D=0.01$, and $E=0.5$. (a) $N V$ interface $(T=0.211)$. The equilibrium tilt angle is $\Psi_{\mathrm{eq}}=0^{\circ}$ (as for nematogens in the $n$ CB series [3-5]); the interface is uniaxial. (b) $N I$ interface $(T=0.225)$. The equilibrium tilt angle is $\Psi_{\text {eq }}=32.5^{\circ}$, i.e., the director is oblique tilted with respect to the normal to the interface (as for nematogens of the $n \mathrm{CB}$ series $[10,11])$. 
that, within our simple model, the parameter $E$ must be positive in order to predict a tilted orientation at the interface. The orientational transition may then be described as the result of a competition between orientations dictated by different terms in the expansion of $u_{\text {ani }}$.

Approximations based on the model described by Eqs. (5) and (6) that neglect the variation of the density and order parameter profiles in the interfacial region, such as the sharp-kink approximation, yield an expression for the surface tension that may be cast in the form

$$
\gamma(\Psi)=\gamma_{0}+\gamma_{2} P_{2}(\cos \Psi)+\gamma_{4} P_{4}(\cos \Psi),
$$

where the coefficients $\gamma_{0}, \gamma_{2}$, and $\gamma_{4}$ depend on the intermolecular potential parameters $(A-E)$, as well as on the bulk values of $\rho$ and $\eta$ [30]. This expression is identical to the one obtained by the so-called phenomenological theories, where it is derived using symmetry arguments and the coefficients are often arbitrary [35]. It is well known that, in this case, the tilt angle transition form oblique to homeotropic alignment is always continuous, as seen in experiments [2].

We have studied a temperature-driven orientational transition at the $N V$ interface. Since the interfacial effects of the (224) term are much smaller than those of the other terms in the intermolecular potential with $l \neq 0$, in order to observe tilt angles we have to choose values of $C$ and $D$ whose combined effect in determining the surface alignment nearly cancels. We could also have set one of them to zero and chosen the other very small [36]. This, however, seems somewhat less realistic than our particular choice. We have set $\mathrm{C}=0.3, D=0.7$, and $E=0.3$. As discussed in Sec. III C, a system with these values of $C$ and $D$ and with $E=0$, at $T=0.175$, is close to a tilt angle transition $\Psi=0^{\circ} \rightarrow 90^{\circ}$. Under these circumstances, the orientation at the interface far from the triple point is determined by the (224) term, which is proportional to $E$. When $E=0.3$ we find that for $T \leq 0.1945$ a tilted orientation at the interface is obtained (see Fig. 5). At $T=0.195$, the orientation changes discontinuously from tilted to perpendicular. The change in slope of $\gamma(T)$ at this transition is too small to be clearly visible on the scale of Fig. 5. As mentioned previously, the continuous nature of this transition is predicted by most theories, although a first-order transition was previously reported by Kimura and Nakano $[15,17]$. These authors used the sharp-kink approximation, but they included in the intermolecular potential excluded volume effects, which result in additional terms for the surface tension $(18)[15,17]$. These are ultimately responsible for driving the transition to first order. In our case, the first-order nature of the transition results from the coupling of the density and orientational order-parameter profiles in the interfacial region. It seems likely that our theory may also predict continuous orientational transitions for different sets of parameters $(C, D$, and $E)[15,17]$. We have not investigated this possibility, but very recently Braun et al. [37] have indeed observed a continuous transition using this theory for a system with $D=0$ (and $C \neq 0$ and $\mathrm{E} \neq 0)$.

The sharp-kink approximation used by Sullivan and

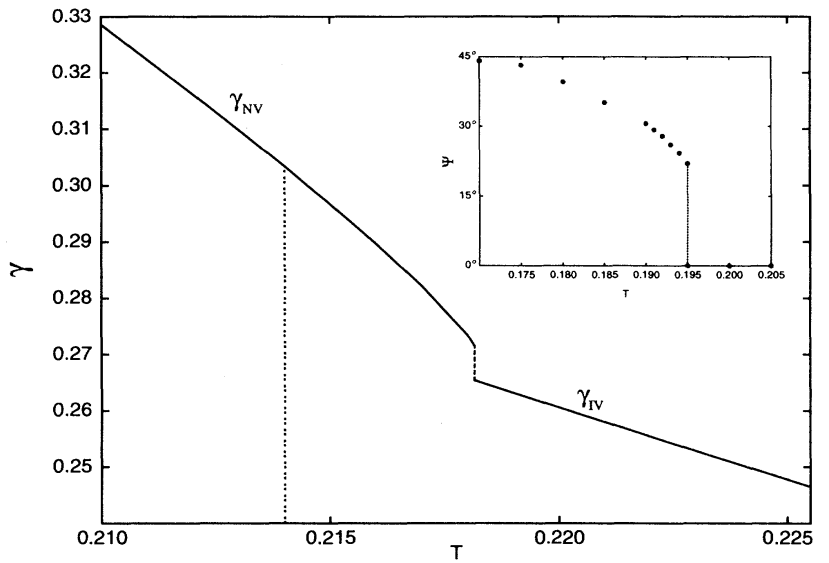

FIG. 5. Liquid-vapor surface tension $\gamma$ versus temperature $T$ (in units of A) close to the triple point $T_{\mathrm{tr}}$. For $T<T_{\mathrm{tr}}$, the surface tension corresponds to the $N V$ interface, while for $T>T_{\mathrm{tr}}$, it corresponds to the $I V$ interface. The discontinuity in the surface tension at $T=T_{\mathrm{tr}}$ is equal in magnitude to the surface tension of the $N I$ interface, indicating that the $N V$ interface is wet by the isotropic phase. The first-order transition from tilted to homeotropic alignment occurring at $T \approx 0.195$ is shown in the inset. The second tilt angle transition, which takes place at $T \approx 0.214$, is indicated by the dotted line.

Tjipto-Margo predicts (for these values of the parameters) a continuous transition from tilted to perpendicular alignment, at a temperature above the triple point, i.e., in contrast to the results of the full theory there is no orientational transition at the $N V$ interface for a system with $C=0.3, D=0.7$, and $E=0.3$.

When the temperature is increased towards the triple point, the $N V$ interface of this system exhibits a (second) orientational transition from perpendicular to planar alignment. This transition is also of first order and occurs at $T=0.214$, which is very close to the triple point temperature $\left(T_{\mathrm{tr}}=0.2182\right)$, suggesting that it may be related to a wetting transition at the $N V$ interface. This observation is further supported by the fact that, for this system, the equilibrium orientation at the $N I$ interface (close or at the triple point) is $\Psi=90^{\circ}$. An inspection of Fig. 5 shows that the surface tension is no longer linear with temperature for $T>0.214$. This departure from linear behavior is due to an increase of the interfacial disorder as expected in a regime of wetting by the isotropic phase. The discontinuity in the surface tension, at the triple point, is equal to $\gamma_{N I}$, confirming that the $N V$ interface is wet by the $I$ phase at that temperature. In the next subsection we will discuss other wetting related phenomena at the $N V$ and at the isotropic liquid-vapor $(I V)$ interfaces.

\section{E. Wetting transitions}

Tijpto-Margo et al. [24] have studied the wetting behavior of this model with $D=E=0$. At the triple point 
temperature $T_{\mathrm{tr}}$, the $N V$ interface is wet by the $I$ phase for values of $C$ in the range $-0.1175-0.0552$. At these points, the $N V$ interface undergoes first-order transitions from partial to complete wetting by $I$. As the absolute value of $C$ increases so does the tendency of the nematic phase to wet the $I V$ interface, until at $C=0.8477$ and $C=-2.1249$ first-order wetting transitions of the $I V$ interface by $N$ occur.

Thus we fix $C=0.9$ and investigate the wetting behavior of the $I V$ interface by varying $D$. First, we set $D=0.15$ and $E=0$. We found that for this value of $D$ the system exhibits partial rather than complete wetting by $N$. The dewetting induced by $D$ is related to the fact that $D>0$ favors a molecular orientation that is parallel to the interface, while $C>0$ favors homeotropic alignment. For these values of the parameters, the effect of $C$ dominates and the molecular orientation is perpendicular at both the $N V$ and $N I$ interfaces. The $N I$ and $N V$ surface tensions increase with $D$ more rapidly than the $I V$ surface tension; consequently, for sufficiently large values of $D$ the $I V$ interface becomes nonwet (i.e., a transition from complete to partial wetting occurs).

The results of Sec. III B indicate that an orientational transition should occur at the $N I$ interface for a value of $D$ close to 0.15 . In order to clarify the relationship between the dewetting transition at the $I V$ interface and the orientational transition at the $N I$ interface we have set $C=0.9$ and $E=-0.6$ and varied the value of $D$. This choice of $E$ favors the state of complete wetting by the $N$ phase. $E<0$ favors the same orientation at the interface as $C>0$; indeed, for this choice of $E$ the value of $D$ required to destroy the complete wetting state increases. A weak first-order transition from total to partial wetting by the $N$ phase occurs, in this case, at $D=0.1162$ (see Fig. $6)$.

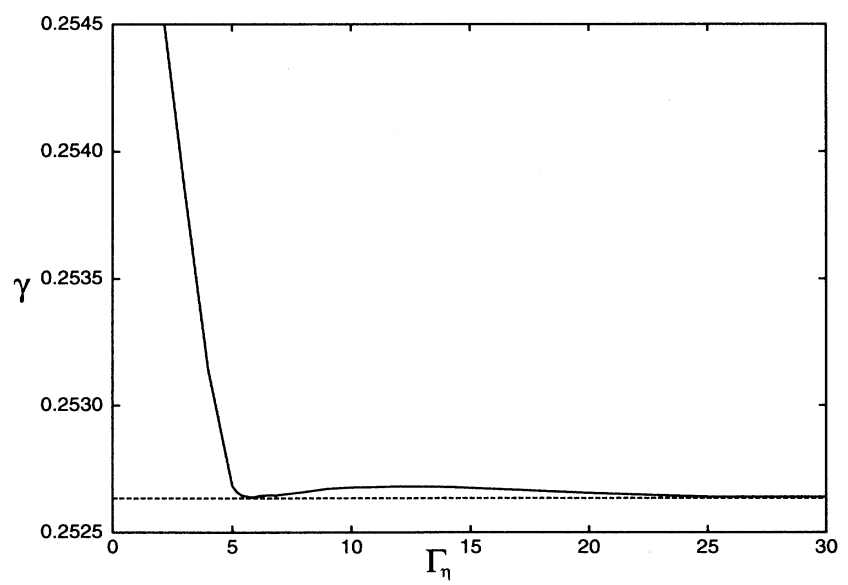

FIG. 6. Excess grand potential $\gamma$ as a function of the adsorption $\Gamma_{\eta}$ of the order parameter $\eta$ at the wetting transition, which ocurrs for $D=0.1162$ when $C=0.9$ and $E=-0.6 . \Gamma_{\eta}$ is defined as $\Gamma_{\eta}=\int_{-\infty}^{\infty}\left[\eta(z)-\eta_{\text {bulk }}\right] d z$ (where $\eta_{\text {bulk }}$ is the bulk nematic order parameter for $z<0$ and zero otherwise). The two minima have the same depth and correspond to the thin and thick nematic wetting films, which coexist at the transition. Note the existence of a small energy barrier indicating a weak first-order transition.
For a larger value of $D$, an orientational transition occurs at the $N I$ interface (from $\Psi=0^{\circ}$ to $\Psi=90^{\circ}$ ) at $T=T_{\mathrm{tr}}$. For a system with $D<0.16, \Psi=0^{\circ}$, while for $D>0.17, \Psi=90^{\circ}$. Notice that the orientational transition at the $N I$ interface takes place for a value of $D$ corresponding to the partial wetting regime of the $I V$ interface. For this set of parameters the tilt angle at the $N V$ interface is $\Psi=0^{\circ}$. Following an argument of Sullivan and Lipowsky [38], competing orientations at the $N I$ and $N V$ interfaces induce an elastic long-ranged repulsive interaction between them, which favors the growth of an $N$ layer at the $I V$ interface. For this system and within our numerical calculations we have been unable to verify this prediction, i.e., the $I V$ interface remains nonwet for a range of parameters where the $N I$ and $N V$ interfaces exhibit different preferred orientations. We think that within this (limited) range of parameters the shortranged (nonelastic) interactions dominate and a state of partial wetting of the $I V$ interface is found. By a judicious choice of parameters the dewetting transition may be made to occur at a value of $D$ closer to the value corresponding to the tilt angle transition of the $N I$ interface. Under these circumstances, the elastic forces may be effective in restoring the state of complete wetting. These numerical calculations, however, are extremely delicate and time consuming and thus we have not investigated the possibility of reentrant wetting transitions induced by elastic forces.

As far as the wetting behavior at the $I V$ interface is concerned, we conclude that, in general, the inclusion of terms that enhance the preferred molecular orientation at the $N I$ and $N V$ interfaces favors the wetting state. Likewise, the inclusion of terms that favor different orientations at the $N$ interfaces will tend to destroy the state of complete wetting and if sufficiently strong will induce a dewetting transition. Finally, complete wetting of the $N V$ interface by the $I$ phase is also favored by the inclusion of terms that compete in determining the surface alignment since these have a disordering effect that favors a wetting transition by the $I$ phase.

\section{CONCLUDING REMARKS}

We have presented a detailed study of the orientational effects induced at the $N I$ and $N V$ interfaces of a model liquid crystal. The anisotropic interactions, which are attractive on average, were assumed to be given by a truncated expansion in spherical harmonics (up to secondorder terms) and the system was studied in the context of a mean-field theory for nonuniform fluids. Although this model is still a highly idealized representation of real liquid crystal interactions, we believe that it captures some of the relevant features that determine the orientational order at nematic interfaces.

We have confirmed an earlier result obtained using a simplified theory $[28,30]$ that quadrupole-quadrupole interactions are crucial in order to account for oblique molecular alignment at the nematic interface. This contribution is embodied in the (224) term with $E>0$. The experimental observations of different molecular align- 
ments at the $N V$ and $N I$ interfaces of a given nematogen have also been described as the result of a competion between the (202) and (222) terms in the anisotropic intermolecular potential. Neither of these effects was properly accounted for by previous related models [23,24] since they include only one term with $l \neq 0$ [namely, the (202) term] in the intermolecular potential. One significant result of this work is that temperature-driven orientational transitions can be found in systems characterized by the potential of Eqs. (5) and (6). It is worth mentioning that Kimura and Nakano $[15,17]$ concluded that no such transitions may occur if only attractive interactions are considered. We note, however, that the attractive interactions used in Refs. $[15,17]$ differ in an essential way from Eqs. (5) and (6), since Kimura and Nakano [15,17] have not included the (202) term (there are also differences in the models used for the hard-core repulsions, but they are of no relevance for this discussion). It is easily shown [28], within the sharp-kink approximation used in Refs. $[15,17]$, that the $(202)$ term is responsible for the temperature dependence of the tilt angle when the anisotropic potential is given by Eqs. (5) and (6). It is unclear to us why Kimura and Nakano $[15,17]$ have not included this term in their attractive potential.

For a given choice of parameters, we have found an orientational transition, that is related to a wetting transition of the $N V$ interface. As the temperature increases towards the triple point, an isotropic film grows at the $N V$ interface, becoming macroscopic at $T_{\mathrm{tr}}$. As the molecular orientations at the $N V$ and $N I$ interfaces in this system are different, the orientation at the $N V$ interface changes to that of the $N I$ interface as the film thickens.

Finally, it was found that complete wetting by the $N$ phase may be destroyed as a result of the competition between the (202) and (222) terms. Similarly, a reentrant wetting transition by the $I$ phase was found when the ratio $D / C$ increases (for $C D>0$ ).

\section{ACKNOWLEDGMENTS}

We are grateful to D. E. Sullivan for useful discussions and for Ref. [28]. We would also like to thank P. I. C. Teixeira for a critical reading of the manuscript. This work was supported in part by DGICYT of Spain through Project No. PB91-0602 and by JNICT of Portugal through Project No. PBIC/C/CEN/1844/92. One of us (E. M. R.) would like to acknowledge Junta de Andalucía (Spain) for financial support.
[1] K.E. Gubbins, in Fluid Interfacial Phenomena, edited by C. A. Croxton (Wiley, New York, 1986), Chap. 10.

[2] B. Jérôme, Rep. Prog. Phys. 54, 391 (1991).

[3] M.G.J. Gannon and T.E. Faber, Philos. Mag. A 37, 117 (1978).

[4] J.E. Proust and L. Ter-Minassian-Saraga, J. Phys. (Paris) Colloq. 40, C3-490 (1979).

[5] H. Yokoyama, S. Kobayashi, and H. Kamei, Mol. Cryst. Liq. Cryst. 99, 39 (1983).

[6] M.A. Bouchiat and D. Langevin, Phys. Lett. 34A, 331 (1971).

[7] P. Chiarelli, S. Faetti, and L. Fronzoni, Lett. Nuovo Cimento 36, 60 (1983).

[8] D. Langevin and M.A. Bouchiat, Mol. Cryst. Liq. Cryst. 22, 317 (1973).

[9] N.V Madhusudana and K.R. Sumathy, Mol. Cryst. Liq Cryst. 92, 179 (1983).

[10] S. Faetti and V. Palleschi, Phys. Rev. A 30, 3241 (1984).

[11] H. Yokoyama, S. Kobayashi, and H. Kamei, Mol. Cryst. Liq. Cryst. 107, 311 (1984).

[12] P. Chiarelli, S. Faetti, and L. Fronzoni, J. Phys. (Paris) 44, 1061 (1983).

[13] P. Chiarelli, S. Faetti, and L. Fronzoni, Phys. Lett. $101 \mathrm{~A}, 31$ (1984).

[14] P.G. de Gennes, Mol. Cryst. Liq. Cryst. 12, 193 (1971).

[15] H. Kimura and H. Nakano, J. Phys. Soc. Jpn. 54, 1730 (1985).

[16] R. Holyst and A. Poniewiersky, Phys. Rev. A 38, 1527 (1988).

[17] H. Kimura and H. Nakano, J. Phys. Soc. Jpn. 55, 4186 (1986).

[18] W.E. McMullen, Phys. Rev. A 38, 6384 (1988).
[19] M. Doi and N. Kuzuu, J. Appl. Polym. Sci.: Appl. Polym. Symp. (U.S.A) 41, 65 (1985).

[20] Z.Y. Chen and J. Noolandi, Phys. Rev. A 45, 2389 (1992).

[21] B.G. Moore and W.E. McMullen, Phys. Rev. A 42, 6042 (1990).

[22] M.M. Telo da Gama, Mol. Phys. 52, 585 (1984).

[23] J.M. Thurtell, M.M Telo da Gama, and K.E. Gubbins, Mol. Phys. 54, 321 (1985).

[24] B. Tjipto-Margo, A.K. Sen, L. Mederos, and D.E. Sullivan, Mol. Phys. 67, 601 (1989).

[25] J.D. Parsons, Mol. Phys. 42, 951 (1980).

[26] P. Harrowell and D.W. Oxtoby, Mol. Phys. 54, 1325 (1985).

[27] M.M. Telo da Gama, in Observation, Prediction and Simulation of Phase Transitions in Complex Fluids, Vol. 460 of Advanced Study Institute, Series C: Mathematical and Physical Sciences: NATO, edited by M. Baus, L.F. Rull, and J.P. Ryckaert (Kluwer, Dordrecht, 1995), pp. 243292.

[28] D.E. Sullivan and B. Tjipto-Margo (unpublished).

[29] C.G. Gray and K.E. Gubbins, Theory of Molecular Fluids (Clarendon, Oxford, 1984).

[30] B. Tjipto-Margo and D.E Sullivan, J. Chem. Phys. 88, 6620 (1988).

[31] P.I.C. Teixeira and M.M Telo da Gama, Mol. Phys. (to be published).

[32] D.E. Sullivan (private comunication).

[33] L. Mederos and D.E. Sullivan, Phys. Rev. A 39, 854 (1989).

[34] P. Tarazona and R. Evans, Mol. Phys. 48, 799 (1983).

[35] T.J. Sluckin and A. Poniewiersky, in Fluid Interfacial 
Phenomena, edited by C. A. Croxton (Wiley, New York, 1986), Chap. 5.

[36] P.I.C. Teixeira and T.J. Sluckin, J. Chem. Phys. 97, 1498 (1992).
[37] F.N. Braun, T.J. Sluckin, E. Velasco, and L. Mederos (unpublished).

[38] D.E. Sullivan and R. Lipowsky, Can. J. Chem. 66, 553 (1988). 